\title{
Green Supply Chain Management Optimization Based On NSGA-II Method
}

\author{
S. Sundar, C. Dhanasekaran, S. Sivaganesan
}

\begin{abstract}
Green Supply Chain Management (GSCM) is the adopted by many companies due to the government policies of various countries. The optimization technique can be applied in the GSCM to increase the profit of the company. In this research, Non-dominated Sorting Genetic Algorithm-II (NSGA-II) technique is applied for the optimization of GSCM to increase the performance. The NSGA-II method has the advantage of choosing the solution closer to the pareto-solution and uses the elitist technique to preserve the best solution in the next generation. Mathematical model of the GSCM system is established and data is provided as input to the mathematical mode. Data is generated in three types, small scale, medium scale and large scale. The proposed NSGA-II method has high performance in the optimization technique compared to existing method. The proposed NSGA-II method has the Number of Pareto Solution (NPS) metrics of 17 for large scale data, while existing method has 14.
\end{abstract}

Index Terms: Green Supply Chain Management, Non-dominated Sorting Genetic Algorithm -II, Elitist technique, Mathematical model, and Number of Pareto Solution.

\section{INTRODUCTION}

Government regulations or public environment awareness has enforced the companies to apply Green Supply Chain Management (GSCM) and green innovation. Both practices are important to apply in the companies to improve the environmental factors [1]. In this scenario, the management of companies are focusing on the GSCM to increase the efficiency in the process [2]. In recent years, many companies are enabling the GSCM to ensure the environment regulation in the supply chain. Supply chain members are need to co-operate with one another to make the efficient contracts [3]. Although great progress has been made related to the drivers of GSCM and several issues remain unsolved [4]. Optimization method is need to be applied to increase the efficiency and to maintain the economic regulation of the companies [5]. The economic criteria are not enough to

Revised Manuscript Received on December 30, 2019.

* Correspondence Author

S. Sundar*, Department of Mechanical Engineering, Vels Institute of Science, Technology and Advanced Studies, Pallavaram, Chennai, India.

Dr. C. Dhanasekaran, Department of Mechanical Engineering, Vels Institute of Science, Technology and Advanced Studies, Pallavaram, Chennai, India.

Dr. S. Sivaganesan, Department of Mechanical Engineering, Vels Institute of Science, Technology and Advanced Studies, Pallavaram, Chennai, India.

(C) The Authors. Published by Blue Eyes Intelligence Engineering and Sciences Publication (BEIESP). This is an open access article under the CC BY-NC-ND license (http://creativecommons.org/licenses/by-nc-nd/4.0/) evaluate the supplier and various perspective should be taken into consideration [6].

The DEMATEL method is developed that involves in analysis of the different factor in supply chain including environmental concerns [7]. Industry consists of many dynamic parameters such as cost and potential market demand. As these parameters were varying in the market and to be analyzed efficiently. Therefore, supply chain management are need to consider to solve the uncertainty problem. Fuzzy logic is one method that come closer to reality in the GSCM. Several methods have been developed to apply fuzzy method to solve the uncertainty problem in GSCM [8 - 9]. Although some researchers have made an attempt to examine the barriers of GSCM and has limitations in their analytical models [10]. In this research, the NSGA-II optimization technique is applied in the GSCM. The NSGA-II method has the advantages of preserving the best solution in the next generation and choosing the solution closer to pareto-solution. The experimental result shows that the performance of the developed method is high compared to other techniques.

The paper is organized as latest research in GSCM is provided in the section II, the proposed method explanation is provided in section III, the experiment result analysis in three scale factors are provided in section IV and conclusion is provided in section $\mathrm{V}$.

\section{LITERATURE REVIEW}

Government regulations enforces the companies around the globe to move towards the GSCM techniques. The existing method involves in the GSCM were reviewed in this section for understanding the recent method.

Wang, et al. [11] analysis the several factor act as the barrier in the GSCM system in Indian food companies. The method proposed Decision Making Trial and Evaluation Laboratory (DEMATEL) for find the key barrier in the system. The experimental analysis provides the clarity for the packaging industries in the understanding of major barriers. The experimental result shows that the aspects like lack of adequate training and progress monitor is reason for the lack of adoption of GSCM in the industries. The optimization method can be applied to increase the performance of the method. Sari, et al. [12] applied the decision framework to analyze the practice of the GSCM. The framework is depending on the combination of Monte Carlo Simulation and Analytical Hierarchy Process and VIKOR method in fuzzy environment.

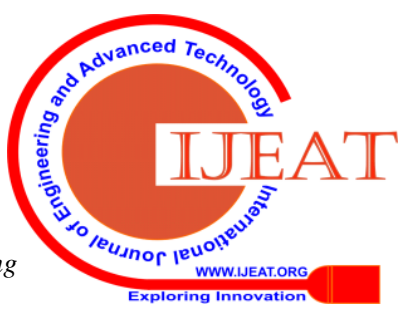


The factors of GSCM is weighted based on the analytical hierarchy process and ranking process is done by VIKOR model depend on performance. This method can be used to recommend the best performing organization in GSCM technique. The incomplete or missing information of the GSCM can be dealt using Monte Carlo Simulation. The optimization method can be used to improve the performance and efficient statistical model to increase the analysis.

Malviya and Kant, [13] proposed hybrid method for the GSCM based on the DEMATEL and fuzzy multi-criterial decision making based on the framework and analyze the success/failure of GSCM model. The fuzzy DEMATEL metrics were used to analyze each evaluation criteria and analyze the success of GSCM. The rank method is applied in the aspects of the GSCM and analyze the correlation among them. The experimental analyze of the developed method is applied in the gear manufacturing organization and evaluated its performance. The result shows that the developed method solves the complex problem. The efficiency of the method is need to be improved.

Kaur, et al. [14] presented DEMATEL based method to analyze the barriers in the Canadian context. The data of the various manufacturing firms from electronics goods sectors were collected. The experimental analysis shows that the three major barriers were involves in the GSCM of Canadian sectors. The barriers of lack of economic awareness, lack of training and monitoring. The developed research helps in support for the Canadian firms. The optimization techniques can be applied to increase the performance of analysis.

Zaid, et al. [15] analysis the link between the GSCM and the Human resource management bundle practices. Along with that the impact of triple bottom lines of sustainability performance system. The customized survey of 121 firm data of pollutant manufacturing sectors in Palestine was used to analyze the performance of the developed method. The experimental result of the analysis shows that the GSCM practice has the positive impact in the sustainable performance in the method. The good performance analytical model can be applied to increase the performance of the method.

Govindan, et al. [16] proposed hybrid optimization technique based on Particle Swarm Optimization (PSO), Electromagnetism Mechanism Algorithm (EMA), and Artificial Bee Colony (ABC) and each is hybridized with Variable Neighborhood Search (VNS) for the optimization. The data are generated with three scale and analyzed using five metrics in the optimization process. The developed hybrid model is compared with hybrid of Genetic Algorithm and VNS to analyze the performance. The experimental analysis shows that the proposed hybrid method has high performance than existing method. The hybrid VNS method provides the less number of solution and this can be overcome by storing the best solution in the next generation. The solution provided by the VNS are far from the pareto-solution.

In order to overcome the limitation of the above existing method in GSCM, the proposed method is applied.

\section{PROPOSED METHOD}

The block diagram of the proposed NSGA-II method in the multi-objective optimization of Green Supply chain management, is shown in Fig. 1.

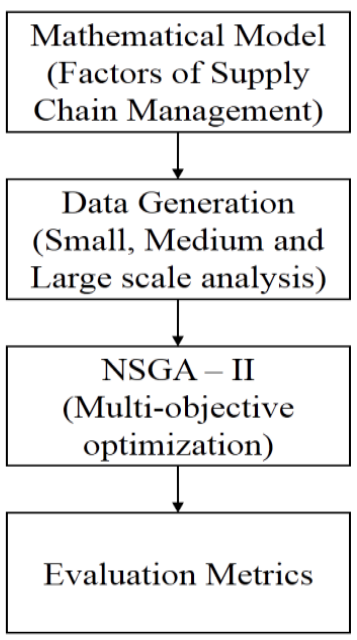

Fig. 1. The block diagram of NSGA-II method in Green Supply chain management

Major strategic decisions in the supply chains is involves in selecting the manufactures location and potential sites Distribution Centers (DCs), suitable technology selection and retailer's allocation. Operational decisions are involving in routing the vehicles from manufacturers to DCs and scheduling vehicles based on time window. Each vehicle serves as a subset of DC's and return to its original manufacturer.

This paper aims to optimize the model decision variables based on the suitable metaheuristic method in the view of three pillars of sustainability. As economic performance is important in the supply chain management, the total cost is considered as a distinct objective; environmental and social impacts are combined together for other objective.

\section{A. Mathematical Model}

Mathematical model of the relationship between the factors in the firms are developed based on the research [16]. In this problem, consider (i) the number and retailer's location is set and each is assigned with only one DC; (ii) retailers demand must be satisfied; (iii) the number of vehicles $K$ are set, with the standard workday of maximum time capacity ( $W$ ); (iv) soft-time window are considered; (v) each layer (retailer, DC and manufacturer) has the number and location of facilities are set; (vi) there is no product flow between the facilities placed in a common echelon.

\section{B. Multi-objective optimization}

The algorithms developed for single objective often stops when it achieves single optimal solution and multi-objective algorithm has the tradeoff between two or more parameters, which leads to optimal non-dominated solutions named as Pareto-optimal solution. For each of Pareto-optimal solution, one objective is satisfied with the cost of affecting another. Multi-objective optimization requires a posterior setting over objectives by the decision maker.

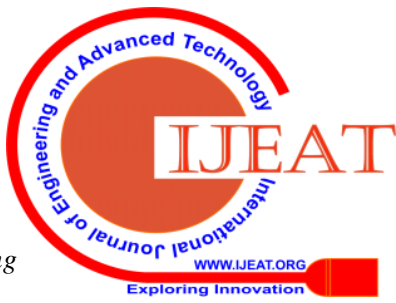


The scalarization is the common technique to solve the problem of multi objective problem. This method involves in applying the single objective method for different objective and selects the optimal value for all the user defined objectives. The formula for single objective space for a minimization problem is shown in Eq. (1).

$\left[\sum_{i=1}^{2}\left(\frac{f_{i}(x)-f_{i}^{*}}{f_{i}^{*}}\right)^{p}\right]^{1 / p}$

Where $f^{*}$ represents the minimum value of $i^{\text {th }}$ objective and this is used to solve the problem of various objective. More emphasis is dedicated to the large deviation by increasing the exponent $p$. Finding the appropriate value of exponent is the challenging task in the method. The developed method provides a single Pareto solution based on the decision maker settings. The aforementioned method with the weighting method is combine to generate a Pareto front [16], given in Eq. (2).

$\left[\sum_{i=1}^{2} w_{i}\left(\frac{f_{i}(x)-f_{i}^{*}}{f_{i}^{*}}\right)^{p}\right]^{1 / p}$

Where $w_{i}$ is the weight associated that must satisfy as follows as in Eq. (3).

$$
\sum_{i=1}^{2} w_{i}=1
$$

The Pareto frontier will be generated by changing these weights.

\section{NSGA II - Multi objective optimization}

A heuristic algorithm namely "Elitist Non-dominated Sorting Genetic Algorithm" (NSGA II) is proposed to solve this problem.

As seen in the previous sub-section, the multi-objective optimization method provides the set of points known as Pareto optimal solution to provide trade-off between the different objective. Number of techniques were proposed to obtain the Pareto-optimal solutions as in evolutionary algorithms. Among these, NAGA II is considered as the powerful method to solve the multi objective problem. This method is developed from the well-known optimization method namely Genetic Algorithm and this is applied with non-dominated sorting concept. In past decades, NSGA II techniques have been widely applied to various design optimization problems. In this research, the NSGA is applied to solve multi objective optimization technique in the GSCM for three generated scale of industries. The mathematical model is used to provide the estimation of the cost and emission based on the parameter. The description of the NSGA II algorithm is presented below:

1). Set initial population $P_{0}$ of size $N$.

2). Develop an offspring population $Q_{t}$ based on the binary tournament selection using crossover, mutation and crowding comparison operator performed on the parent population $\left(P_{t}\right)$, where subscript " $t$ " represent the number of generations. The parent population and offspring population are combined to provide entire population $R_{t}$.

3). In the entire population, perform non-dominated sorting method to identify the non-dominated fronts of objective functions $F_{1}, F_{2} F_{1}, F_{2}$ etc.

4). Developed a new parent population $(P t+1)$ of size $N$ from the obtained fronts $\left(F_{i}\right)$.

5). Continue the process until the maximum iteration is reached.

\section{EXPERIMENTAL RESULTS}

The optimization technique in supply chain management helps to reduce the cost and increase eco-friendly process. The hybrid algorithms were proposed in the supply chain management for various factors to increase the performance. In this research, NSGA-II is proposed in GSCM for the multi-objective optimization. To evaluate the performance of the developed method, data were generated. The evaluation metrics were applied to analyze the performance of the developed method. The developed method is tested with the three hybrid algorithm in the GSCM management [16] i.e., HPV, HEV, HAV. The developed method is compared with existing method depend on obtained pareto front.

Simulation Condition: The developed method is evaluated in the system consists of $1.4 \mathrm{GHz}$ of intel i5 process, 8 GB RAM and 500 GB hard disk.

\section{A. Data generation}

The numerical experiment was conducted to evaluate the performance of the developed method. The problem of numerical experiment was constructed with various size of the firms such as Manufacturer (M), potential distributor centers (D), Vehicles (V), Retailers (R), Products (G), and Potential Technologies (T) is shown in table (). Based on the facilities of each stage, the problem is classified into three types: small, medium and large. The Normal Distribution $\mathrm{N}$ $(50,10)$ was used to generate the demand of the retailers. The factors of the data are generated based on the research [16].

\section{B. Evaluation Metrics}

To evaluate the quality of non-dominated solution provided by aforementioned method, different performance metrics were considered. The metrics used to measure the performance of the proposed NSGA-II method is given below:

1). Number of Pareto Solutions (NPS) - Number of total Non-dominated solutions obtained by an algorithm (More is better).

2). Percentage of Domination (POD) - analysis the algorithm ability in dominating the solution of other algorithm (More is better).

3). Data envelopment Analysis (DEA) - analyze the efficiency of method against other algorithms.

\section{Performance Analysis}

The proposed NSGA-II method is compared with the existing method in the optimization of GSCM. Data are generated in the three classification such as small scale, medium scale and large scale.

Published By:

Blue Eyes Intelligence Engineering 


\section{Green Supply Chain Management Optimization Based On NSGA-II Method}

\section{a. Small Scale data}

The proposed NSGA-II method is analyzed in the small scale data and compared with existing method. The evaluation metrics were calculated from the method for the optimization of GSCM.

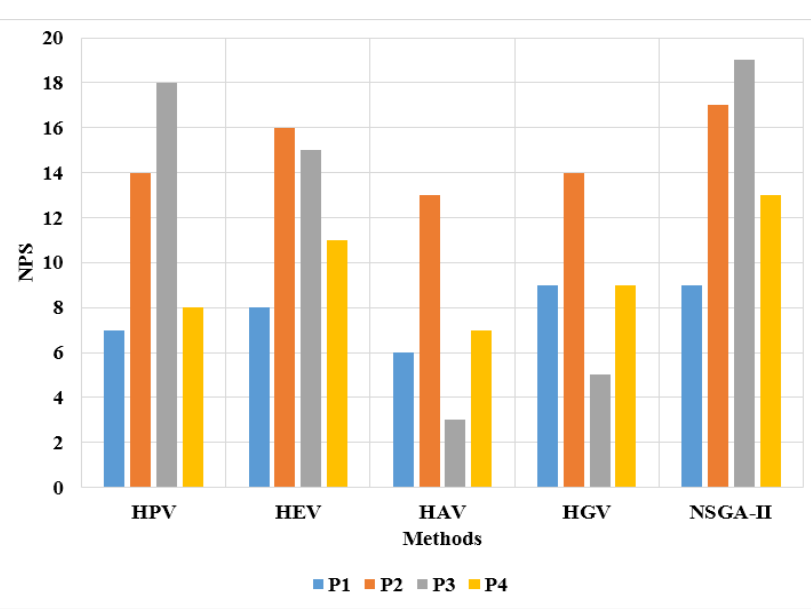

Fig. 2. NPS metrics in optimization

The NPS metrics is measured for the developed method and compared with other existing methods such as HPV, $\mathrm{HEV}, \mathrm{HGV}$ in small scale data, as shown in Fig. (2). This shows that the proposed NSGA-II method has the higher performance compared to other methods. The proposed NSGA-II method has the NPS metrics of 17 for problem 2, while existing HEV method has 16 . The developed method provides the more number of solution in the method, which increase the NPS metrics.

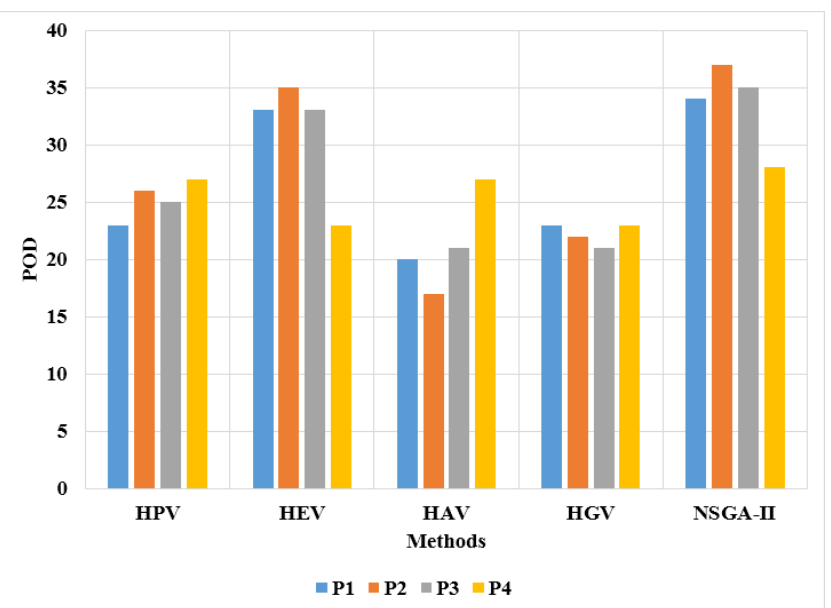

Fig. 3. POD of the optimization

The POD metrics were measured from the performance of the proposed NSGA-II method and compared with other existing method, as shown in Fig. (3). This shows that the proposed NSGA-II method has the higher performance compared with other techniques. The NSGA-II method has the advantage of using elitist technique to preserve best solution of the current population. The developed method has the higher performance for four problems.

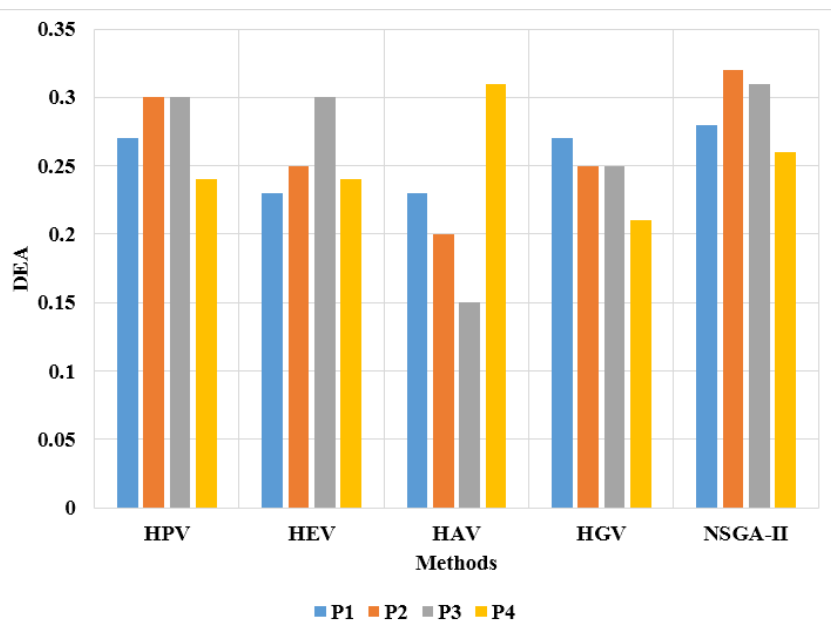

Fig. 4. DEA metrics in small scale data

The DEA metrics were evaluated from the proposed NSGA-II method and compared with existing method, as shown in Fig. (4). The proposed NSGA-II method has the higher performance compared to other existing method. The proposed NSGA-II method has the higher performance for four problems in the method. Therefore, the proposed NSGA-II method has the higher performance for the small scale data.

\section{b. Medium scale data}

The data are generated in medium scale and analyze the performance of the proposed NSGA-II method. The four problems were created to analyze the efficiency of the optimization technique.

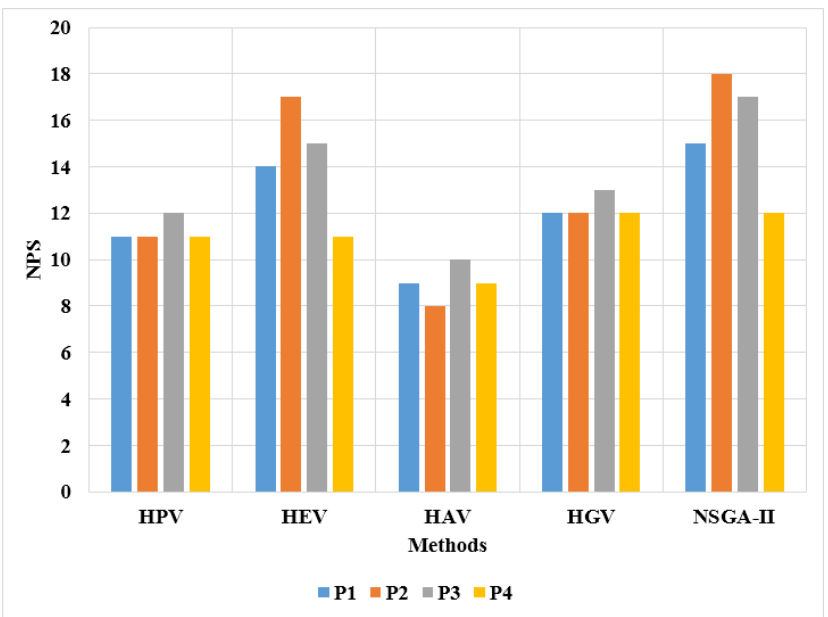

Fig. 5. NPS Metrics in medium scale data

The NPS metrics is measured for the proposed NSGA-II method for medium scale data and compared with existing method, as shown in Fig. (5). This shows that the proposed NSGA-II method has the higher performance compared with other techniques. The proposed NSGA-II method provides more solution for the data and this increase the NPS metrics. 


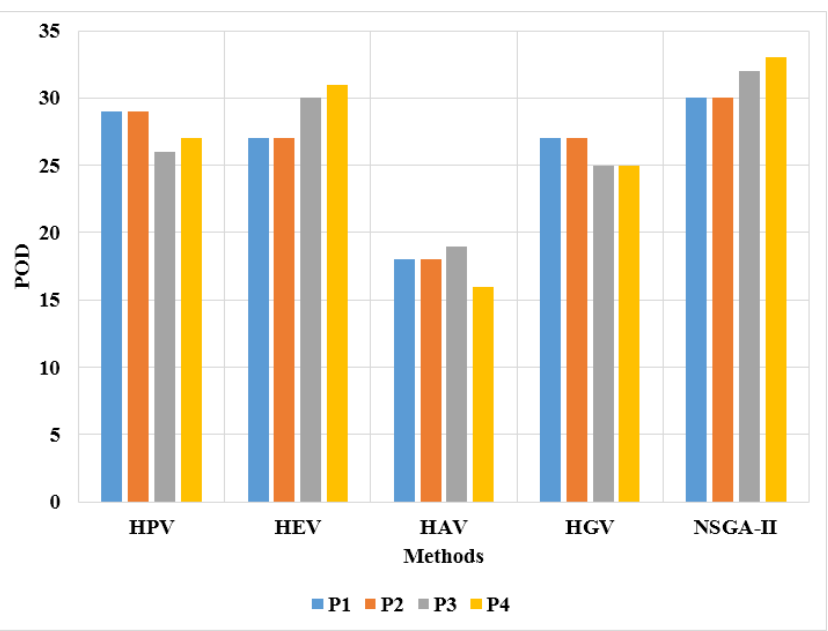

Fig. 6. POD metrics for medium scale data

The POD metrics is measured for the medium scale data of the proposed NSGA-II method and compared with existing method of HAV, as shown in Fig. (6). This shows that the proposed NSGA-II method has the higher performance compared with other existing methods. The proposed NSGA-II method has the finds the dominance solution due to the method provides the solution as close as pareto-optimal solution.

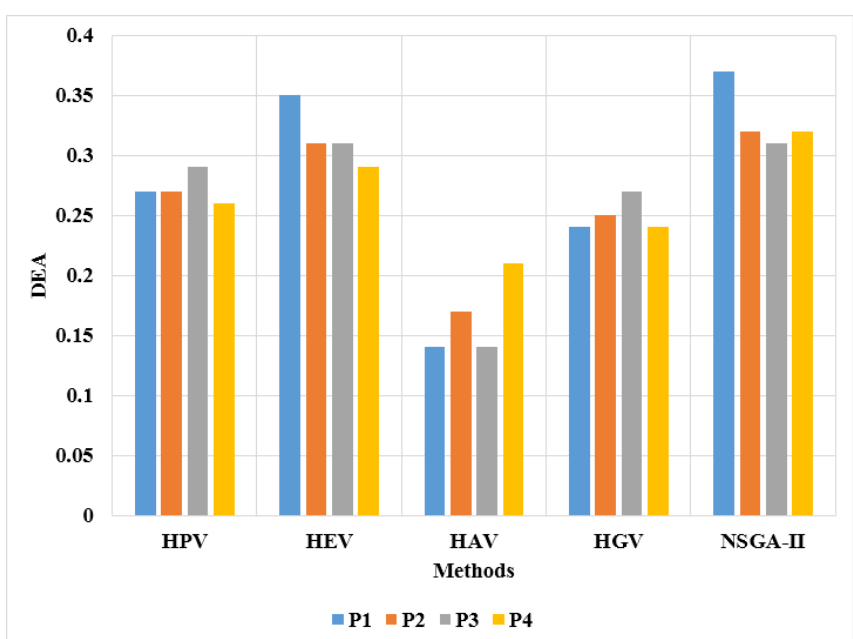

Fig. 7. The DEA metrics for medium scale data

The existing method and developed method is analyzed with the DEA metrics in the medium scale data and compared with each other, as shown in Fig. (7). The proposed NSGA-II method has the higher performance compared to other existing methods. The proposed method has the advantages of preserving the best solution of the current population in the next generation. The performance of the proposed NSGA-II method is high in the medium scale data.

\section{c. Large scale data}

The proposed NSGA-II method is evaluated in the large scale data to analyze the performance.

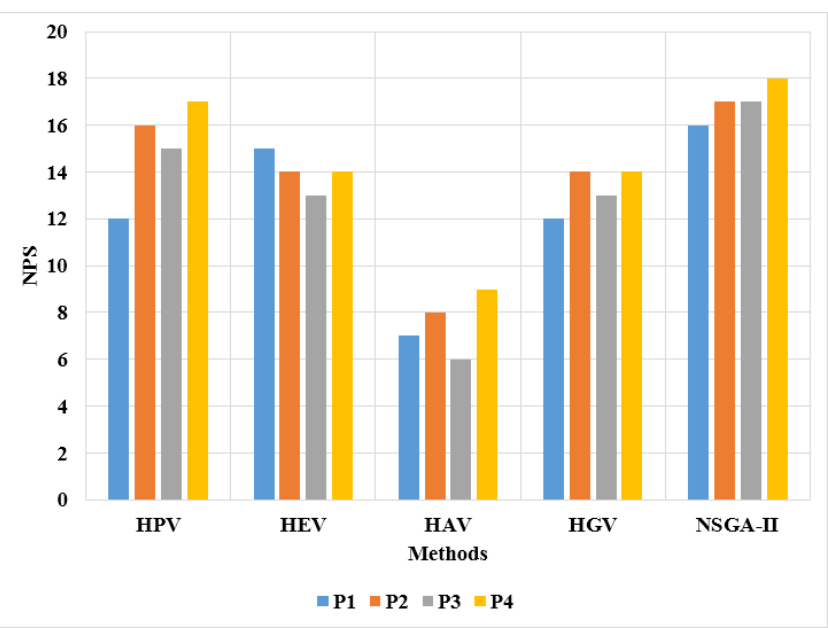

Fig. 8. The NPS metrics for large scale data

The NPS metrics is measured for the proposed NSGA-II for four kinds of problems in the large scale data in GSCM, as shown in Fig. (8). The proposed NSGA-II method is compared with existing method and this shows that the proposed NSGA-II method has the higher performance than other methods. The NPS metrics is high for the proposed NSGA-II method due to number of solution provided by the NSGA-II method is high. The NSGA-II method provides more number of solution because the best solution is stored in the next generation. The proposed NSGA-II method has the NPS of 18 for problem twelve, while existing method HPV has 17 solutions.

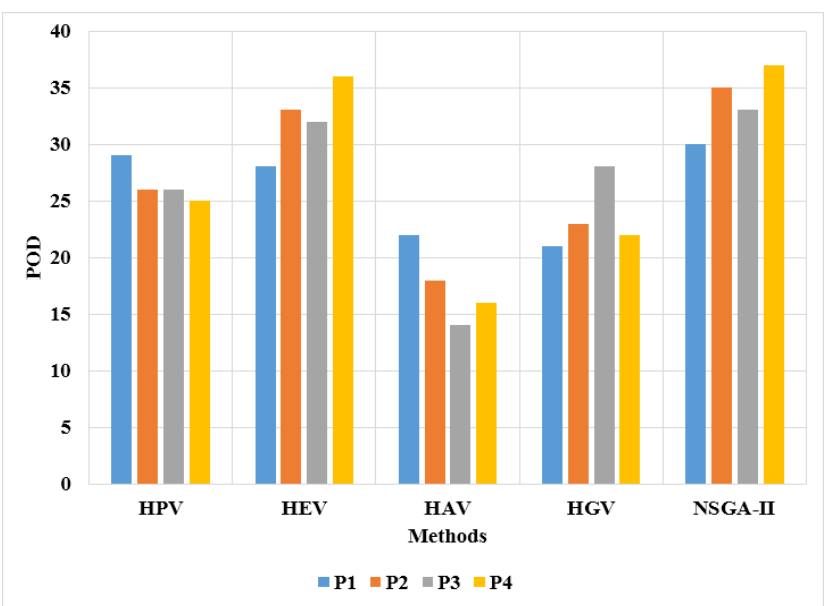

Fig. 9. The POD metrics for large scale data

The POD metrics is measured for the proposed NSGA-II method and compared with existing method, as shown in Fig. (9). The proposed NSGA-II method has the higher performance compared with the existing method. The proposed NSGA-II method has the advantage of selecting the solution closer to pareto-solution. The proposed NSGA-II has the POD of 37, while existing HEV method has the POD of 36 . 


\section{Green Supply Chain Management Optimization Based On NSGA-II Method}

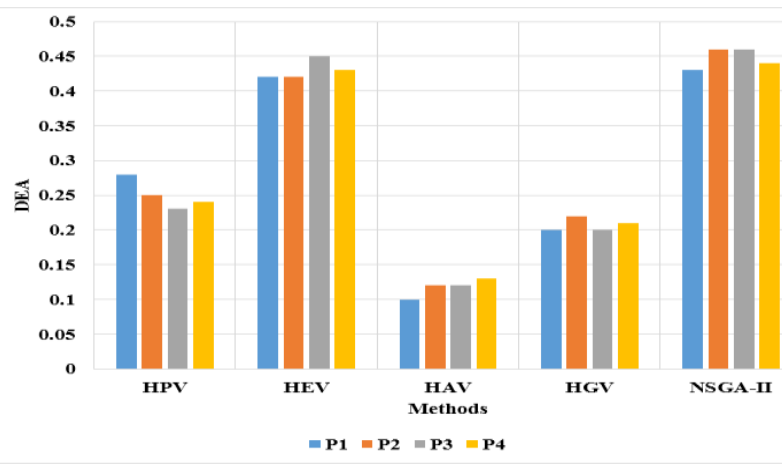

Fig. 10. The DEA metrics for the large scale data

The DEA metric is measured for proposed NSGA-II method and compared with existing method, as shown in the Fig. (10). The proposed NSGA-II method uses the elitist technique to preserve the solution. The proposed NSGA-II method has the DEA of 0.44 , while existing HEV method has 0.43. Therefore, the proposed method has the higher performance in the optimization of GSCM compared to existing techniques. The proposed method is evaluated with various metrics, which shows the proposed NSGA-II method higher performance in multi-objective optimization.

\section{CONCLUSION}

Hybrid optimization technique is applied in the GSCM system and has the lower performance due to limited number of pareto-solution. In this research, the NAGO-II optimization technique is applied in the GSCM mathematical model. The proposed and existing method is tested with three scale data to analyze the performance. The proposed NSGA-II method has the higher performance compared to other existing method. The proposed NSGA-II method has the advantage of preserving the best solution using elitist technique and choosing the solution closer to pareto-solution. The experimental result shows that the proposed NSGA-II method has higher performance in three metrics. The NPS of the proposed method has 17 solutions, while existing method has 14 solutions. The future work of the developed method is need to evaluate in the real time data.

\section{REFERENCES}

1. N. A. A. Seman, K. Govindan, A. Mardani, N. Zakuan, M. Z. M. Saman, R. E. Hooker, and S. Ozkul. (2019). The mediating effect of green innovation on the relationship between green supply chain management and environmental performance. Journal of Cleaner Production, 229. pp. 115-127.

2. M. A. Sellitto, F. F. Hermann, A. E. Blezs Jr, and A. P. Barbosa-Póvoa. (2019). Describing and organizing green practices in the context of Green Supply Chain Management: Case studies. Resources, Conservation and Recycling. 145. pp. 1-10.

3. J. Noh, and J. S. Kim. (2019). Cooperative green supply chain management with greenhouse gas emissions and fuzzy demand. Journal of cleaner production, 208. pp.1421-1435.

4. Z. Wang, Q. Wang, S. Zhang, and X. Zhao. (2018). Effects of customer and cost drivers on green supply chain management practices and environmental performance. Journal of Cleaner Production, 189. pp. 673-682.

5. Y. Li, and K. Mathiyazhagan. (2018). Application of DEMATEL approach to identify the influential indicators towards sustainable supply chain adoption in the auto components manufacturing sector. Journal of cleaner production. 172. pp. 2931-2941.

6. H. G. Gören. (2018). A decision framework for sustainable supplier selection and order allocation with lost sales. Journal of Cleaner Production. 183. pp. 1156-1169.

7. B. B. Gardas, R. D. Raut, and B. Narkhede. (2018). Evaluating critical causal factors for post-harvest losses (PHL) in the fruit and vegetables supply chain in India using the DEMATEL approach. Journal of cleaner production. 199. pp. 47-61.

8. A. Mohammed. R. Setchi, M. Filip, I. Harris, and X. Li. (2018). An integrated methodology for a sustainable two-stage supplier selection and order allocation problem. Journal of Cleaner Production. 192. pp. 99-114.

9. K. Mathiyazhagan, U. Datta, A. Singla, and S. Krishnamoorthi. (2018). Identification and prioritization of motivational factors for the green supply chain management adoption: case from Indian construction industries. Opsearch. 55(1). pp. 202-219.

10. M. A. Moktadir, S. M. Ali, R. Rajesh, and S. K. Paul. (2018). Modeling the interrelationships among barriers to sustainable supply chain management in leather industry. Journal of cleaner production. 181. pp. 631-651.

11. Z. Wang, K. Mathiyazhagan, L. Xu, and A. Diabat. (2016). A decision making trial and evaluation laboratory approach to analyze the barriers to Green Supply Chain Management adoption in a food packaging company. Journal of Cleaner Production, 117. pp. 19-28.

12. K. Sari. (2017). A novel multi-criteria decision framework for evaluating green supply chain management practices. Computers \& Industrial Engineering. 105. pp. 338-347.

13. R. K. Malviya, and R. Kant. (2016). Hybrid decision making approach to predict and measure the success possibility of green supply chain management implementation. Journal of Cleaner Production. 135. pp. 387-409.

14. J. Kaur, R. Sidhu, A. Awasthi, S. Chauhan, and S. Goyal. (2018). A DEMATEL based approach for investigating barriers in green supply chain management in Canadian manufacturing firms. International Journal of Production Research. 56(1-2). pp. 312-332.

15. A. A. Zaid, A. A. Jaaron, and A. T. Bon. (2018). The impact of green human resource management and green supply chain management practices on sustainable performance: An empirical study. Journal of cleaner production, 204. pp.965-979.

16. K. Govindan, A. Jafarian, and V. Nourbakhsh. (2019). Designing a sustainable supply chain network integrated with vehicle routing: a comparison of hybrid swarm intelligence metaheuristics. Computers \& Operations Research. 110. pp. 220-235.

\section{AUTHORS PROFILE}

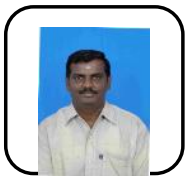

S. Sundar is working as an Assistant Professor in the Department of Mechanical Engineering, Dr. MGR Educational and Research Institute, Chennai. He has completed B.E (Mechanical Engineering) from University of Madras and also completed M.E (Industrial Engineering) from Anna university. He has also completed Master of Business Administration (MBA) with specialization in Marketing from IGNOU.

At present he is pursuing $\mathrm{PhD}$ in Vels Institute of Science, Technology and Advanced Studies, Pallavaram, Chennai, India. He has 25 years of teaching experience. He is specialized in Engineering Graphics, TQM, Financial Management He has attended various Faculty development programmes.

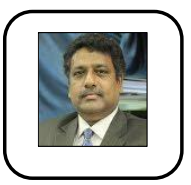

Dr. C. Dhanasekaran is working as a Professor in the Department of Mechanical Engineering, Vels Institute of Science, Technology and Advanced Studies, Pallavaram, Chennai, India. He completed his PhD in Anna University and he had 25 Years of teaching experiences. Dr C. Dhanasekaran has over 20 publications, two engineering Text Books and he had a patent in "Apparatus and method for welding". He is specialized in IC Engine, Alternate fuels, Heat transfer and Composite materials and he is member of various national and international Professional bodies.

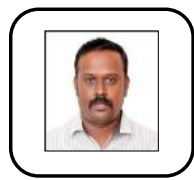

Dr. S. Sivaganesan is working as an Associate Professor in the Department of Mechanical Engineering, Vels Institute of Science, Technology and Advanced Studies, Pallavaram, Chennai, India. He completed his $\mathrm{PhD}$ in VELS University and he had 13 Years of teaching experiences. He is active member of various national and international Professional bodies. He has over 30 publications. He is specialized in I.C. Engines, Alternate fuels, PCM and Composite materials.

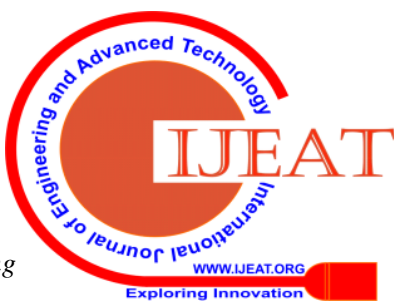

